J. Lake Sci. (湖泊科学), 2022, 34(1): 142-150

DOI 10. 18307/2022. 0113

(C) 2022 by Journal of Lake Sciences

\title{
南极湖泊沉积物中有机硫组成及其与铁硫化物的联系”
}

\author{
沈丽丽,孙婷婷, 郭晓宇, 黄 涛** \\ (安徽大学资源与环境工程学院, 湿地生态保护与修复安徽省重点实验室, 合肥 230601)
}

\begin{abstract}
摘 要: 本文利用有机硫硫同位素组成 $\left(\delta^{34} \mathrm{~S}_{\mathrm{org}}\right)$ 及活性铁的黄铁矿化度 (DOP) 和硫化度 (DOS) 研究了南极阿德雷岛受 企鹅活动影响的 Y2 湖和未受影响的菲尔德斯半岛燕鸥湖 YO 沉积物中有机硫的来源组成及其与铁硫化物的联系. 结果 表明, $\mathrm{Y} 2$ 沉积物 $1 \sim 15 \mathrm{~cm}$ 较低的 $\delta^{34} \mathrm{~S}_{\mathrm{org}}$ 指示有机质硫化形成的贫 ${ }^{34} \mathrm{~S}$ 成岩有机硫 $\left(\mathrm{S}_{\mathrm{diag}}\right.$ ) 占比较高, 其中在 $8 \sim 15 \mathrm{~cm}$ 较高 的 DOS 指示铁硫化物形成和有机质硫化同时发生, 源于该层位高含量的活性铁供给. Y2 剖面 $48 \sim 56 \mathrm{~cm}$ 沉积物有机硫富 集 ${ }^{34} \mathrm{~S}$ 显著, 对应于企我数量高峰期, 表明大量企鹅粪的外部输人使沉积物中富 ${ }^{34} \mathrm{~S}$ 的生物有机硫 $\left(\mathrm{S}_{\text {bio }}\right)$ 快速增加, 并超过 了内部硫转化过程中成岩有机硫的增加. YO 沉积物在 $28 \mathrm{~cm}$ 处 $\delta^{34} \mathrm{~S}_{\mathrm{org}}$ 显著降低, 主要是由于该层位硫酸盐异化还原强, 有机质硫化形成的大量 $\mathrm{S}_{\mathrm{diag}}$ 成为有机硫的主要成分, 同位素质量平衡计算结果显示该层位 $\mathrm{S}_{\mathrm{diag}}$ 占比高达 $95.4 \%$; 同时,28 $\mathrm{cm}$ 处较高的 DOP 指示沉积物黄铁矿的形成与有机质硫化同时发生, 主要源自沉积物中高硫酸盐还原强度产生了足够多 的硫化氢前体. 本研究表明, 有机硫组分的来源转化与有机质、活性铁紧密耦合, 对区域湖泊有机质成岩埋藏及潜在的气 候环境效应具有重要的指示意义.
\end{abstract}

关键词: 硫同位素;硫化度;黄铁矿化度;生物有机硫;成岩有机硫

\section{Organic sulfur compositions and their relationships with iron sulfides in Antarctic lake sedi- ments *}

\section{Shen Lili, Sun Tingting, Guo Xiaoyu \& Huang Tao **}

(Anhui Province Key Laboratory of Wetland Ecosystem Protection and Restoration, School of Resources and Environment Engineering, Anhui University, Hefei 230601, P.R.China)

\begin{abstract}
Sulfur isotope ratios of organic sulfur $\left(\delta^{34} \mathrm{~S}_{\text {org }}\right)$, degrees of pyritization (DOP) and sulfurization (DOS) in sediments were analyzed to reveal the organic sulfur compositions and their relationships with iron sulfides in a penguin-affected lake Y2 and a pristine Yanou Lake ( YO) at Ardley Island and Fildes Peninsula, Antarctica, respectively. The depleted $\delta^{34} \mathrm{~S}_{\text {org }}$ values in Y2 sediments of $1-15 \mathrm{~cm}$ indicated a high proportion of ${ }^{34} \mathrm{~S}$-depleted diagenetic organic sulfur $\left(\mathrm{S}_{\text {diag }}\right)$ in total sulfur. Meanwhile, the high DOS in Y2 sediments of 8-15 cm indicated a concurrent sulfurization of iron and the organic matter, likely due to the high availability of active iron in this layer. The observed much enriched $\delta^{34} \mathrm{~S}_{\text {org }}$ in $\mathrm{Y} 2$ sediments of $48-56 \mathrm{~cm}$ corresponding to the period of high penguin populations, which indicated that large external inputs of penguin guanos result in a rapid increase in ${ }^{34} \mathrm{~S}$-enriched biological organic sulfur $\left(S_{\text {bio }}\right)$ in the sediments, and exceeds the $S_{\text {diag }}$ that from the internal sulfur transformations. The depleted $\delta^{34} \mathrm{~S}_{\text {org }}$ in YO sediment of $28 \mathrm{~cm}$ indicates a high proportion $(95.4 \%)$ of ${ }^{34} \mathrm{~S}$-depleted $\mathrm{S}_{\text {diag }}$ which was from strong sulfurization of organic matter, corresponding to the strongest sulfate reduction in this layer. Meanwhile, the high DOP in YO sediment of $28 \mathrm{~cm}$ indicated a concurrent formation of pyrite sulfur and the sulfurization of organic matter in this layer, due to the high available precursors of hydrogen sulfide and/or intermediate species of sulfur from the sulfate reduction. Our study indicates that the components of organic sulfur are coupled tightly with organic matter and reactive iron and provides a direct implication for the depositions of organic matter and thus the potential climatic and environmental effects in regional aquatic ecosystems.
\end{abstract}

Keywords: Sulfur isotope ratios; degree of sulfurization; degree of pyritization; biological organic sulfur; diagenetic organic sulfur

* 2021-03-27 收稿;2021-05-10 收修改稿.

国家自然科学基金项目 (41476165) 和安徽省高校自然科学研究重点项目 (2019KJ0042) 联合资助.

** 通信作者;E-mail: huangt@ ahu.edu.cn. 
水域生态系统中硫的转化与有机质成岩埋藏、营养元素循环、水体酸化、重金属生物有效性等紧密耦 合, 产生重要的环境效应 ${ }^{[1-4]}$. 有机硫 (organic sulfur, $\mathrm{S}_{\text {org }}$ ) 包括由硫酸盐同化还原形成的生物有机硫 $\left(\mathrm{S}_{\mathrm{bio}}\right)$ 和 由有机质硫化形成的成岩有机硫 $\left(S_{\text {diag }}\right)$, 是湖泊沉积物中硫的主要赋存形态之一, 占比可高达 $90 \%{ }^{[5-6]}$. 生 物有机硫和成岩有机硫中的还原态硫功能团均可络合 $\mathrm{Hg}^{+} 、 \mathrm{Cu}^{2+} 、 \mathrm{~Pb}^{2+} 、 \mathrm{Ag}^{+}$及 $\mathrm{As}^{3+}$ 等金属离子, 对有毒有害 元素的形态和生物有效性具有重要的抑制作用 ${ }^{[7]}$. 成岩有机硫的形成可提高活性有机质的稳定性及其在沉 积物中的埋藏保存, 而且硫化过程记录了氧化还原条件和元素生物地球化学循环等重要的环境信息 ${ }^{[8]}$. Sinninghe Damsté 等 ${ }^{[9]}$ 较早提出有机质成岩埋藏的自然硫化机制, 即 $\mathrm{S}^{2-}$ 优先与铁结合形成铁硫化物, 当 $\mathrm{S}^{2-}$ 含 量超过铁硫化物计量配比时, 便与有机质结合硫化形成有机硫, 这主要发生在有机质早期成岩阶段. 湖泊沉 积物中的活性有机质在好氧和戻氧条件下可降解形成温室气体二氧化碳和甲烷 ${ }^{[10]}$, 因此, 开展硫转化及其 与有机质结合、调控的相关研究对认识区域碳、硫循环及潜在的气候环境效应具有重要意义.

活性铁是指能被 $1 \mathrm{~mol} / \mathrm{L}$ 盐酸提取的铁,主要包括无定形或弱晶型 $\mathrm{Fe}$ ( III) 氧化物和 $\mathrm{Fe}$ ( II ) (黄铁矿除 外) 以及 $\mathrm{Fe}_{3} \mathrm{~S}_{4}$ (含量很少, 可忽略不计) ${ }^{[11]}$. 活性铁与硫化物可发生铁的硫化作用, 生成酸可挥发性硫 ( acid volatile sulfur, AVS) 和黄铁矿硫 (pyrite sulfur, CRS). AVS 和 CRS 是沉积物中还原性无机硫 (RIS) 的两个主 要终产物 ${ }^{[12-14]}$, 它们的形成会抑制有机质硫化, 而高含量活性铁对 CRS 的形成和有机质硫化则具有促进作 用, 使得有机质硫化受制于 C-S-Fe 耦合体系 ${ }^{[15]}$. 黄铁矿化度 (DOP) 和硫化度 (DOS) 是评估沉积物中黄铁矿 和铁硫化物的形成是受有机质还是活性铁控制的重要指标 ${ }^{[16-17]}$. DOP 值是表征沉积物氧化还原条件并识别 黄铁矿形成的限制因素 ${ }^{[18-19]}$, 其判断标准为 DOP $>0.75$ 为缺氧环境, $0.45<\mathrm{DOP}<0.75$ 为贫氧环境, $\mathrm{DOP}<0.45$ 为氧化环境 ${ }^{[20]}$. 由于自然界中存在大量的 AVS, DOP 并不能完全指征铁与硫化物反应的程度, 而 DOS 可以 更好地表征铁对硫化物的限制程度.

硫同位素组成被广泛应用于水域生态系统中硫的来源及其转化循环过程研究 ${ }^{[8,21-23]}$, 在解析有机硫来 源组成及其转化方面具有重要作用 ${ }^{[15,24]}$. 沉积物中有机硫相对于同时存在的硫化物富集 ${ }^{34} \mathrm{~S}$ 约为 $10 \%$, 但在 某些条件下可达 $30 \%$ 以上 ${ }^{[25-27]}$, 这主要与有机硫中生物有机硫和成岩有机硫的形成途径有关. 硫酸盐同化 还原过程导致的硫同位素分馏效应仅为 $1 \% 0 \sim 3 \%{ }^{[28]}$, 形成的生物有机硫与其前体硫酸盐具有相似的硫同位 素组成; 而硫酸盐异化还原生成的硫化氢及其氧化/歧化产物中间态硫的 ${ }^{34} \mathrm{~S}$ 亏损显著 ${ }^{[8,23,29-30]}$, 成岩有机硫 由硫化氢/中间态硫与有机质化合形成,其硫同位素组成继承前体硫化氢/中间态硫偏负的信号. 总有机硫 硫同位素组成取决于其生物有机硫和成岩有机硫的组成比例,介于硫化物和硫酸盐之间 ${ }^{[31]}$. 因此, 硫同位 素是研究有机硫来源组成、转化以及成岩有机硫与铁硫化物联系的重要手段.

南极菲尔德斯半岛和阿德雷岛位于全球变暖最快的南极半岛西北部, 随着冰川后退、陆地出露和雪冰 消融, 沿海岛屿形成了众多湖泊和集水区; 其中, 阿德雷岛一些湖泊接受了企鹅活动带来的大量有机质和营 养元素 ${ }^{[32]}$, 成为温室气体重要的排放源. 我们前期对阿德雷岛受企鹅活动影响的 Y2 湖和未受影响的菲尔 德斯半岛燕鸥 (YO) 湖沉积物中硫和重金属形态组成的分析研究 ${ }^{[33-34]}$ 表明, 有机硫是 Y2 湖沉积物中硫的主 要赋存形态, 企鹅活动带来的高含量有机质驱动了强烈的硫酸盐还原, 进而限制了微量重金属的生物有效 性. 而有关 Y2、YO 湖沉积物有机硫来源的具体组成及其与有机质、铁硫化物的联系等还不清楚. 因此,本研 究在前期工作的基础上,利用总有机硫硫同位素组成、活性铁的 DOP 和 DOS 解析并揭示南极湖泊有机硫的 来源组成及其与铁硫化物间的联系, 对认识湖泊硫生物地球化学转化及其与有机质成岩埋藏、重金属生物 有效性的关联提供了视角, 并为进一步开展南极气候变化敏感区湖泊硫转化的气候环境效应研究奠定了 基础.

\section{1 材料与方法}

\section{1 研究区域与样品采集}

南极菲尔德斯半岛和阿德雷岛位于乔治王岛西南部, 面积约 $33 \mathrm{~km}^{2}$, 是乔治王岛最大的无冰区, 也是中 国南极科学考察站长城站所在地. 当地气候寒冷, 年平均气温为 $-2.2^{\circ} \mathrm{C}$. 菲尔德斯半岛和阿德雷岛上的大部 分湖泊是由冰川活动形成的, 其中阿德雷岛 $\mathrm{Y} 2$ 湖位于该岛西部, 为一小型月牙状淡水湖, 面积约 $3500 \mathrm{~m}^{2}$, 水深在 $0.5 \mathrm{~m}$ 左右, 海拔高度约 $12 \mathrm{~m}$, 南侧邻海, 地势东高西低. Y 2 湖周边历史时期有企鹅活动, 湖泊沉积物 
中含有大量企鹅转移而来的有机质、营养盐和重金属污染物 ${ }^{[32]}$; 而菲尔德斯半岛的 YO 湖位于中国南极长 城站东南方向, 为一现代小型湖泊, 面积约 $9000 \mathrm{~m}^{2}$, 距离长城湾最近的海岸线约 $200 \mathrm{~m}$; $\mathrm{YO}$ 湖未受企鹅活动 影响, 营养元素含量较低.

本研究分析的 Y2、YO 湖沉积物为 Chen 等 ${ }^{[33]}$ 研究的平行样. 样品于中国第 29 次南极科学考察期间 (2012-2013 年) 分别采集于南极阿德雷岛的 Y2 湖和菲尔德斯半岛的 YO 湖 (图 1). 企我粪土沉积剖面 Y2 按 $1 \mathrm{~cm}$ 间隔分成 60 个样, 原生湖泊沉积剖面 $Y O$ 按 $1 \mathrm{~cm}$ 间隔分成 30 个样. 在进行化学分析之前, 一部分 样品 $-20^{\circ} \mathrm{C}$ 冷冻保存, 而另一部分样品则在去除岩石碎片和生物残体后冷冻干燥并研磨过篮 ( 孔径为 0.125 $\mathrm{mm})$. 本文选择 Chen 等 ${ }^{[33]}$ 硫形态研究中的 16 个 Y2 和 11 个 YO 沉积物序列进行分析.

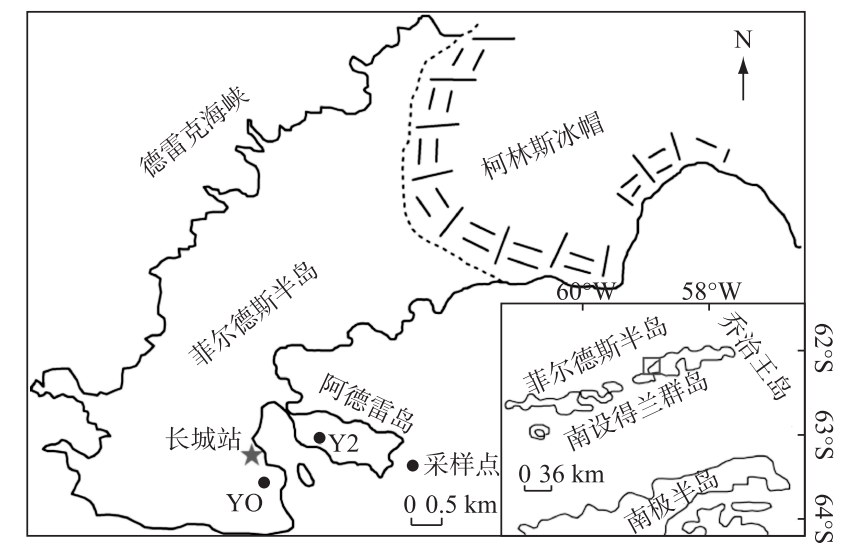

图 1 南极半岛乔治王岛阿德雷岛和菲尔德斯半岛的研究区和采样点 (参照 Chen 等 ${ }^{\left[{ }^{[3]}\right.}$ )

Fig.1 Study area and sampling sites on Fildes Peninsula and Ardley Island according to Chen et al ${ }^{[34]}$

\section{2 样品分析与处理}

1.2.1 活性铁分析 在氮气手套箱内称取解冻沉积物 $0.5 \mathrm{~g}$, 加人 $25 \mathrm{~mL} 1 \mathrm{~mol} / \mathrm{L} \mathrm{HCl}$, 在室温下振荡 $24 \mathrm{~h}$, 然 后离心 ( $6000 \mathrm{r} / \mathrm{min}, 15 \mathrm{~min})$. 吸取 $25 \mathrm{~mL}$ 上清液置于 $100 \mathrm{~mL}$ 雉形瓶中, 加人 $1 \mathrm{~mL}$ 盐酸 $(1: 3)$ 和 $1 \mathrm{~mL}$ 质量 分数为 $10 \%$ 的盐酸差胺, 煮沸至 $15 \mathrm{~mL}$, 冷却至室温后转移至 $100 \mathrm{~mL}$ 容量瓶并定容, 混匀后移取 $1 \sim 5 \mathrm{~mL}$ 于 $50 \mathrm{~mL}$ 比色管中定容, 使用邻菲啰啉分光光度法测定提取液中总活性铁 $\mathrm{Fe}(\mathrm{HCl})$ 的含量 ${ }^{[19]}$, 实验室内样品 重复测定误差小于 $5 \%$.

1.2.2 DOP 和 DOS 的计算 黄铁矿化度 $(\mathrm{DOP})$ 和硫化度 $(\mathrm{DOS})$ 按下列公式 ${ }^{[35]}$ 计算:

$$
\begin{gathered}
\mathrm{DOP}=\mathrm{Fe}(\mathrm{CRS}) /[\mathrm{Fe}(\mathrm{CRS})+\mathrm{Fe}(\mathrm{HCl})] \\
\operatorname{DOS}=[\mathrm{Fe}(\mathrm{AVS})+\mathrm{Fe}(\mathrm{CRS})] /[\mathrm{Fe}(\mathrm{CRS})+\mathrm{Fe}(\mathrm{HCl})]
\end{gathered}
$$

式中, $\mathrm{Fe}$ ( CRS) 为黄铁矿结合态铁的含量, 按 $\mathrm{FeS}_{2}$ 的 $1: 2$ 化学计量比 $\mathrm{CRS} / 2$ 计算; $\mathrm{Fe}(\mathrm{HCl})$ 为总活性铁含 量; $\mathrm{Fe}(\mathrm{AVS})$ 为假定 AVS 全部以 $\mathrm{FeS}$ 形式存在的硫化物结合态 $\mathrm{Fe}$ 的含量; AVS 和 CRS 含量数据引自 Chen 等 $^{[33]}$.

1.2.3 有机硫硫同位素分析 前期研究中利用 $\mathrm{NaH}_{2} \mathrm{PO}_{4}$ 溶液和改进的冷扩散法依次提取了沉积物中的 $\mathrm{SO}_{4}^{2-}, \mathrm{AVS} 、 \mathrm{CRS}$ 和单质硫 (elemental sulfur, ES $)^{[33]}$. 提取后的 AVS 和 CRS 中加人足量 $\mathrm{AgNO}_{3}$ 溶液转化为 $\mathrm{Ag}_{2} \mathrm{~S}$, 用于硫同位素分析 ${ }^{[36]}$. 本研究将提取了 $\mathrm{SO}_{4}^{2-} 、 \mathrm{AVS} 、 \mathrm{CRS}$ 和 $\mathrm{ES}$ 后的残余沉积物用于有机硫硫同位素样 品制备. 沉积物用蒸馏水洗涤 3 次, 然后转移至烧杯中烘干、研磨; 取定量粉末样与其 3 倍质量的艾氏卡试 剂 (质量比轻质 $\mathrm{MgO}: \mathrm{Na}_{2} \mathrm{CO}_{3}=2: 1$ ) 混合均匀, 置于瓷坩埚中并覆盖一层艾氏卡试剂; 将坩埚置于马弗炉中 $800^{\circ} \mathrm{C}$ 焙烧 $2 \mathrm{~h}$, 样品中有机硫全部转化为硫酸钡; 待样品冷却后, 加 $300 \mathrm{~mL}$ 蒸馏水加热 $30 \mathrm{~min}$ 溶解并经 0.2 $\mu \mathrm{m}$ 滤膜过滤; 用 $\mathrm{HCl}$ 调节滤液 $\mathrm{pH}<2.0$ (防止铁水解), 加 $10 \mathrm{~mL} 10 \% \mathrm{BaCl}_{2}$, 于 $60^{\circ} \mathrm{C}$ 反应 $12 \mathrm{~h}$ 后过滤、收集 硫酸钡并干燥至恒重. 在矿床地球化学国家重点实验室采用稳定同位素比值质谱仪 (MAT253-Flash EA 2000 ) 测定硫酸钡硫同位素组成, 分析精度为 $0.2 \%$ 。 测试过程中使用 VCDT 标准 IAEA-SO-5 $(+0.5 \% 0)$, 
IAEA-SO-6 $(-34.1 \% 0)$ 和 NBS-127 $(+20.3 \%)$ 作为标样, 结果以 $\delta(\%)$ 表示, 其公式为: $\delta^{34} \mathrm{~S}(\% 0)=\left[\left(\mathrm{R}_{\text {sample }}-\right.\right.$ $\left.\left.\mathrm{R}_{\text {standard }}\right) / \mathrm{R}_{\text {standard }}\right] \times 1000$.

1.2.4 有机硫源占比计算 沉积物中 $\mathrm{S}_{\text {org }}$ 由 $\mathrm{S}_{\mathrm{bio}}$ 和 $\mathrm{S}_{\text {diag }}$ 按一定比例组成, 根据同位素质量平衡按公式 (3) 计算 两种硫源的组成比例.

$$
f \cdot \delta^{34} \mathrm{~S}_{\text {diag }}+(1-f) \cdot \delta^{34} \mathrm{~S}_{\text {bio }}=\delta^{34} \mathrm{~S}_{\text {org }}
$$

式中, $f$ 代表成岩有机硫占总有机硫的比例 $\left(\mathrm{S}_{\mathrm{diag}} / \mathrm{S}_{\text {org }}\right), 1-f$ 代表生物有机硫占总有机硫的比例 $\left(\mathrm{S}_{\mathrm{bio}} / \mathrm{S}_{\text {org }}\right)$; $\delta^{34} S_{\text {diag }}$ 和 $\delta^{34} S_{\text {bio }}$ 分别是成岩有机硫和生物有机硫的硫同位素组成, $\delta^{34} S_{\text {org }}$ 是沉积物总有机硫硫同位素组成. Y2 沉积物中生物有机硫来源于海洋源的企鹅粪, 与海水硫酸盐有相似的硫同位素组成, YO 硫酸盐主要来 自海盐飞沫 ${ }^{[33]}$, 其生物有机硫也具有海水硫酸盐硫同位素组成信号, 即 $\delta^{34} S_{\mathrm{bio}} \approx 20 \%{ }^{[37]}$. 而有机质硫化形成 的成岩有机硫硫同位素继承了硫酸盐异化还原产物硫化氢的硫同位素组成信号, 即 $\delta^{34} \mathrm{~S}_{\mathrm{diag}} \approx \delta^{34} \mathrm{~S}_{\mathrm{AVS}} \approx$ $\delta^{34} \mathrm{~S}_{\mathrm{CRS}}$. 其中, 沉积物 $\delta^{34} \mathrm{~S}_{\mathrm{AVS}}$ 和 $\delta^{34} \mathrm{~S}_{\mathrm{CRS}}$ 数据引自 Shen 等 ${ }^{[36]}$.

\section{2 结果}

$\mathrm{Y} 2$ 和 YO 沉积物 $\delta^{34} \mathrm{~S}_{\text {org }}$ 、活性铁含量、DOP 和 DOS 的垂直分布如图 2 所示. Y2 剖面沉积物有机硫硫同 位素组成呈 3 段式垂直分布特征, 在 1 15 cm 较低 (1.5\% 3.7\% , 平均值为 2.7\%o), 在 19 45 cm 中等且波 动较小 $\left(4.1 \% 0 \sim 5.7 \%\right.$, 平均值为 $4.7 \% 0$ ), 在 $48 \sim 56 \mathrm{~cm}$ 显著富集 ${ }^{34} \mathrm{~S}(8.5 \% 0 \sim 11.4 \%$, 平均值为 $9.6 \% 0)$. $\mathrm{YO}$ 剖 面沉积物 $\delta^{34} \mathrm{~S}_{\mathrm{org}}$ 除 $28 \mathrm{~cm}$ 层位明显偏负外, 从底部到表层呈现波动变负的趋势.

$\mathrm{Y} 2$ 沉积物中总活性铁含量范围为 $15.34 \sim 42.41 \mathrm{mg} / \mathrm{g}$, 平均值为 $25.4 \mathrm{mg} / \mathrm{g}$, 其中在 $8 \sim 19 \mathrm{~cm}$ 含量较高, 其他层位含量平均值为 $21.67 \mathrm{mg} / \mathrm{g}$ 且波动较小. $\mathrm{YO}$ 沉积物活性铁含量为 $4.26 \sim 5.69 \mathrm{mg} / \mathrm{g}$, 平均值为 4.86 $\mathrm{mg} / \mathrm{g}$, 远小于 Y2. Y2 沉积物 DOP $(0.45 \pm 0.21)$ 远低于 DOS $(5.10 \pm 4.13)$,其 DOS 在 $8 \mathrm{~cm}$ 层位达到较高值 (11.82), 在底部 $48 \sim 56 \mathrm{~cm}$ 沉积物的 DOP 和 DOS 均变大且在 $48 \mathrm{~cm}$ 处达到最高值 (12.98). YO 沉积物的 DOP $(0.49 \pm 0.16)$ 和 DOS $(0.83 \pm 0.34)$ 相差不大, 随深度呈波动增大趋势. Y 2 剖面 $1 \sim 8 \mathrm{~cm}$ 沉积物总有机硫 中 $\mathrm{S}_{\mathrm{diag}}$ 占比均大于 $50 \%$, 平均值为 $58.9 \% ; 12 \sim 45 \mathrm{~cm}$ 沉积物 $\mathrm{S}_{\mathrm{diag}}$ 的占比变化不大 $(42.7 \% \sim 56.2 \%)$, 平均值为 $49.9 \%$, 底部 $48 \sim 56 \mathrm{~cm}$ 沉积物 $\mathrm{S}_{\text {diag }}$ 占比均小于 $50 \%$, 均值为 $41.0 \%$. YO 剖面除 $28 \mathrm{~cm}$ 沉积物 $\mathrm{S}_{\text {diag }}$ 占比高达 $95.4 \%$ 外, 其他层位 $\mathrm{S}_{\mathrm{diag}}$ 占比范围为 $39.4 \% \sim 59.2 \%$, 平均值为 $46.2 \%$.

\section{3 讨论}

\section{1 有机硫来源组成}

有机硫根据其形成途径可分为生物有机硫和成岩有机硫 ${ }^{[38-39]}$. 生物有机硫形成于硫酸盐同化还原, 即 生物摄取硫酸盐用于合成氨基酸和其他含硫细胞组分 ${ }^{[36]}$. 企我以粪便的形式将大量生物有机硫输送到 Y2 湖, 形成鸟粪土沉积物 ${ }^{[32]}$. 鸟粪生物有机硫来源于海洋 $\mathrm{SO}_{4}^{2-}$ 的同化还原, 该过程硫同位素分馏可忽略不 计 ${ }^{[28]}$, 故鸟粪生物有机硫与海洋 $\mathrm{SO}_{4}^{2-}$ 有相似的硫同位素组成 $\left(\delta^{34} \mathrm{~S} \approx 20 \%\right.$ ), 企鹅粪便的输人会导致有机硫 显著富集 ${ }^{34} \mathrm{~S}$. 成岩有机硫形成有机质硫化, 即由硫酸盐还原产生的各种还原态硫与有机质结合生成大分子 有机硫化合物, 还原态硫与有机质结合过程硫同位素分馏可忽略, 所以成岩有机硫与 $\mathrm{H}_{2} \mathrm{~S}$ 有相似的 $\delta^{34} \mathrm{~S}$ 值 ${ }^{[31]}$.

$\mathrm{Y} 2$ 剖面 1 15 $\mathrm{cm}$ 沉积物 $\delta^{34} \mathrm{~S}_{\mathrm{org}}$ 值较低, 指示贫 ${ }^{34} \mathrm{~S}$ 的成岩有机硫占比较大. $\delta^{34} \mathrm{~S}_{\mathrm{org}}$ 值在 $8 \mathrm{~cm}$ 处达到最小 值, 表明 $8 \mathrm{~cm}$ 层位沉积物具有最高的有机质硫化程度. Y 2 剖面 $19 \sim 45 \mathrm{~cm}$ 沉积物 $\delta^{34} \mathrm{~S}_{\mathrm{org}}$ 变化不大, 表明该层 位成岩有机硫和生物有机硫组成比例稳定; $\mathrm{Y} 2$ 沉积物 $\mathrm{RIS} / \mathrm{SO}_{4}^{2-}$ 比值指示剖面底部 $48 \mathrm{~cm}$ 硫酸盐还原很 强 ${ }^{[34]}$, 大量的还原态硫易与有机质结合形成硫同位素偏负的成岩有机硫组分, 但由于该层位时段湖泊周边 企鹅种群数量增加 ${ }^{[32]}$, 大量企鹅粪的输人使沉积物中富 ${ }^{34} \mathrm{~S}$ 的生物有机硫也快速增加; 本研究 Y2 剖面 $48 \sim$ $56 \mathrm{~cm}$ 沉积物显著富集的 $\delta^{34} \mathrm{~S}_{\mathrm{org}}$ 指示生物有机硫比例很高, 指示鸟粪带来的生物有机硫超过了由硫酸盐还原 产物硫化氢/中间态硫与有机质形成的成岩有机硫的增加. 此外, 研究表明沉积扰动会加速生物有机硫大分 子有机物快速分解, 形成更多的小分子生物有机硫 ${ }^{[40]}$, 本研究中企我粪便输人沉积的物理扰动过程可能会 

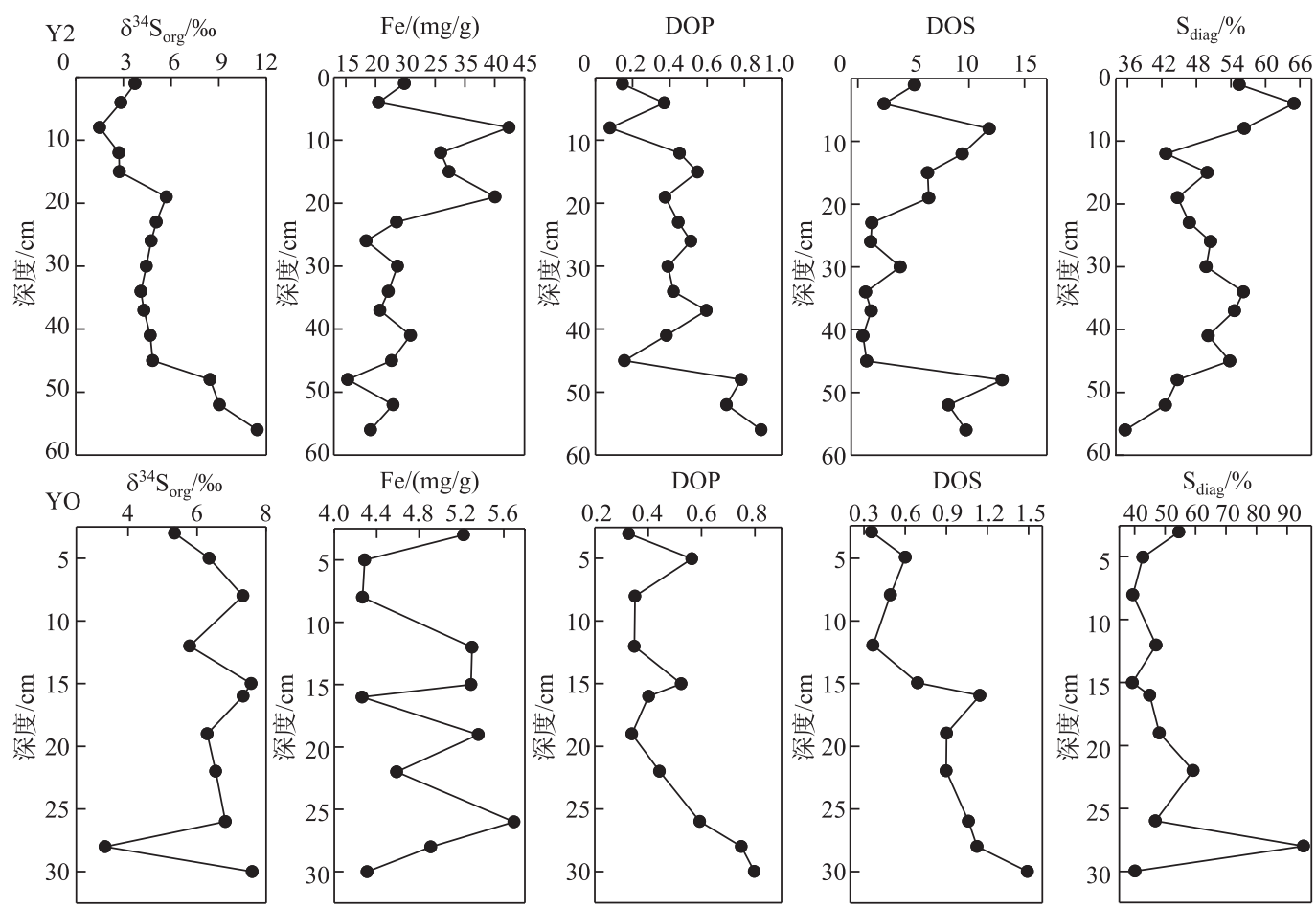

图 $2 \mathrm{Y} 2$ 和 $\mathrm{YO}$ 沉积物中 $\delta^{34} \mathrm{~S}_{\text {org }}$ 活性铁含量、DOP、DOS 和 $\mathrm{S}_{\mathrm{diag}}$ 占比的剖面变化

Fig.2 Vertical changes of $\delta^{34} \mathrm{~S}_{\text {org }}$, reactive iron, DOP, DOS and $\mathrm{S}_{\text {diag }} \%$ in Y2 and YO sediments

产生类似的作用, 分解释放的大量小分子 $\mathrm{S}_{\mathrm{bio}}$ 可快速与有机质有效再结合, 导致沉积物生物有机硫增加.

YO 剖面 $0 \sim 8 \mathrm{~cm}$ 沉积物 $\delta^{34} \mathrm{~S}_{\text {org }}$ 值升高, 与先前 Chen 等 ${ }^{[33]}$ 硫形态指示的明显有机硫矿化相一致, 研究表 明表层沉积物的微量溶解氧能促进微生物分泌一些物质并有利于有机硫的矿化 ${ }^{[41]} ; 8 \sim 26 \mathrm{~cm}$ 和 $30 \mathrm{~cm}$ 层位 沉积物 $\delta^{34} S_{\text {org }}$ 值变化不大, 指示这些层位生物有机硫和成岩有机硫的组成比例稳定. YO 剖面 $28 \mathrm{~cm}$ 处沉积 物 $\delta^{34} S_{\text {org }}$ 值显著降低, 表明成岩有机硫组分显著增加; 前期沉积物硫形态研究指出该层位的硫酸盐还原最 强 ${ }^{[33]}$,产生的硫化氢易与有机质结合形成大量的成岩有机硫.

\section{2 生物有机硫与成岩有机硫的占比}

有机质硫化成岩需要有足量的活性有机质, 有机质不仅参与硫酸盐异化还原产生还原态硫, 还直接与 还原态硫结合形成有机硫 ${ }^{[42-43]}$. Y 2 和 YO 沉积物有机硫中成岩有机硫所占的比例如图 2 所示. Y 2 沉积物 0 8 cm 成岩有机硫所占的比例大于 50\%, 很可能是由于高活性有机质促进了成岩有机质硫化. $48 \sim 56 \mathrm{~cm}$ 沉积物较低的成岩有机硫占比指示了大量的企鹅粪输人和沉积扰动导致生物有机硫占比的增加. YO 沉积 物 $0 \sim 26 \mathrm{~cm}$ 和 $30 \mathrm{~cm}$ 成岩有机硫的比例低于 $50 \%$, 前期研究表明这些层位硫酸盐还原较弱 ${ }^{[33]}$, 较低的硫化 氢供给导致有机质硫化程度较低. YO 沉积物 $28 \mathrm{~cm}$ 成岩有机硫的比例高达 $95.4 \%$, 这是因为 $28 \mathrm{~cm}$ 处硫酸 盐还原强度大 ${ }^{[33]}$, 充足的硫化氢与沉积物中的有机物结合产生大量的成岩有机硫, 其占比大幅增加.

\section{3 有机质硫化与铁硫化的联系}

Y2 剖面沉积物低 DOP 值和高 DOS 值 (图 2) 指示 Y2 容易发生铁的硫化过程; 而 YO 剖面沉积物 DOP> 0.45 , 指示在还原环境中, 大部分活性三价铁氧化物被还原为活性二价铁并有效转化为稳定的 CRS. 目前, 一 些研究表明铁硫化物与成岩有机硫能同时形成 ${ }^{[4-46]}$. 铁氧化物快速输人能提高中间态硫的产生, 而这有可 能同时促进有机质硫化和铁硫化物的形成 ${ }^{[46-48]}$. 本研究中 Y2 剖面沉积物 $8 \sim 15 \mathrm{~cm}$ 的低 $\delta^{34} \mathrm{~S}_{\text {org }}$ 值和高 DOS 指示该层位有机质硫化和铁的硫化同时发生, 表明该层位高含量的活性铁有利于铁硫化物的形成且并促进 了有机质硫化过程; Y2 剖面 48 56 cm 沉积物高 DOS 值是企鹅活动带来的高有机质和强硫酸盐还原促进了 
铁的硫化作用, 铁硫化物 (包括黄铁矿) 的大量形成对有机质硫化具有竞争性抑制作用 ${ }^{[25,42]}$, 从而降低了成 岩有机硫的比例. YO 剖面沉积物 DOP 和 DOS 随深度呈波动增加的趋势, 即深部层位沉积物还原性无机硫 更多以 CRS 为主; 同时, $28 \mathrm{~cm}$ 处强硫酸盐还原提供了充足的硫化氢/中间态硫与有机质结合为成岩有机硫 并降低 $\delta^{34} \mathrm{~S}_{\text {org }}$, 成岩有机硫占比高达 $95.4 \%$, 表明该层位也存在有机质硫化与 CRS 的形成同时发生.

硫在沉积物有机质的成岩和埋藏中发挥着重要作用 ${ }^{[49]}$, 不同形态硫的转化会影响环境变化乃至生态安 全 ${ }^{[50-51]}$. 湖泊硫的转化循环涉及一系列氧化还原反应, 并影响内源磷和铁的迁移释放、有机质的成岩埋藏、 重金属的毒性和生物可利用性, 产生重要的生态环境效应 ${ }^{[17,34,52]}$. 环南极海岛、无冰区分布有众多接收海 鸟、海兽传输的高有机质、营养盐和重金属污染的湖泊和集水区 ${ }^{[53]}$, 这类气候敏感区水生系统水文和氧化还 原条件季节性变化显著, 营养盐生物地球化学转化活跃. 对南极洲沿海地区海兽排泄物的研究中发现, 海兽 排泄物中的有机质具有很高的生物有效性, 在冻融过程中, 有机质会因微生物活性升高发生矿化过程, 使二 氧化碳或甲烷的排放显著增加 ${ }^{[54]}$. 有机质硫化过程能够提高有机质的稳定性, 有效抑制有机质的矿化分 解, 对碳转化循环产生实质影响. 本研究结果表明, 有机硫组分的来源转化与有机质、活性铁紧密耦合, 对区 域湖泊有机质成岩埋藏、铁硫化物形成及其对金属生物有效性限制有直接指示作用, 对进一步开展营养元 素生物地球化学转化的气候环境效应评估具有重要的意义.

\section{4 结论}

1) Y2 剖面底部生物有机硫占比的提高源于大量企鹅粪的输人, 使沉积物中生物有机硫的增加超过了 成岩有机硫; 而 $\mathrm{YO}$ 剖面 $28 \mathrm{~cm}$ 处的成岩有机硫占比的绝对优势源于强硫酸盐还原提供了充足的硫化氢/中 间态硫作为有机质硫化的反应前体.

2) Y2 和 YO 剖面部分层位沉积物有机质硫化与铁的硫化同时发生,主要与高活性铁含量和强硫酸盐 还原过程相关.

致谢: 本文研究样品来源于中国极地沉积物库(合肥).

\section{5 参考文献}

[ 1 ] Yin HB, Fan CX, Ding SM et al. Distribution characteristic and correlation relationship of reactive sulfur and heavy metals in sediments of Meiliang bay and Wuli Lake of Taihu Lake. Environmental Science, 2008, 29(7) : 1791-1796. DOI: 10. 13227/j.hjkx.2008.07.010. [尹洪斌, 范成新, 丁土明等. 太湖梅梁湾与五里湖沉积物活性硫和重金属分布特征及 相关性研究. 环境科学, 2008, 29(7): 1791-1796.]

[ 2 ] Liu J, Jiang T, Huang R et al. A simulation study of inorganic sulfur cycling in the water level fluctuation zone of the Three Gorges Reservoir, China and the implications for mercury methylation. Chemosphere, 2017, 166: 31-40. DOI: 10.1016/j. chemosphere.2016.09.079.

[ 3 ] Zhao YP, Zhang ZQ, Wang GX et al. High sulfide production induced by algae decomposition and its potential stimulation to phosphorus mobility in sediment. Science of the Total Environment, 2019, 650: 163-172. DOI: 10.1016/j. scitotenv. 2018.09.010.

[ 4 ] Shen QS, Fan CX. Identification of black suspended particles in the algae-induced black bloom water column. J Lake Sci, 2015, 27 (4) : 591-598. DOI: 10.18307/2015.0405. [申秋实, 范成新. 藻源性湖泛水体显黑颗粒的元素形态分析 与鉴定. 湖泊科学, $2015,27(4): 591-598$.

[ 5 ] Morgan B, Burton ED, Rate AW. Iron monosulfide enrichment and the presence of organosulfur in eutrophic estuarine sediments. Chemical Geology, 2012, 296/297: 119-130. DOI: 10.1016/j.chemgeo.2011.12.005.

[ 6 ] Wang JF, Chen JG, Dai ZH et al. Sulfur speciation in the surface sediments of lakes from different regions, China: Characterization by S K-edge XANES spectroscopy. Journal of Chemistry, 2016, 2016: 1-9. DOI: 10.1155/2016/3672348.

[ 7 ] Hoffmann M, Mikutta C, Kretzschmar R. Bisulfide reaction with natural organic matter enhances arsenite sorption: Insights from X-ray absorption spectroscopy. Environmental Science \& Technology, 2012, 46 (21) : 11788-11797. DOI: 10. 1021/es302590x.

[ 8 ] Amrani A. Organosulfur compounds: Molecular and isotopic evolution from biota to oil and gas. Annual Review of Earth and Planetary Sciences, 2014, 42(1) : 733-768. DOI: 10.1146/annurev-earth-050212-124126. 
[ 9 ] Sinninghe Damsté JS, Rijpstra WIC, Kock-van Dalen AC et al. Quenching of labile functionalised lipids by inorganic sulphur species: Evidence for the formation of sedimentary organic sulphur compounds at the early stages of diagenesis. Geochimica et Cosmochimica Acta, 1989, 53(6) : 1343-1355. DOI: 10.1016/0016-7037(89)90067-7.

[10] Schink B. Energetics of syntrophic cooperation in methanogenic degradation. Microbiology and Molecular Biology Reviews, $1997,61(2):$ 262-280.

[11] Mortimer RJG, Davey JT, Krom MD et al. The effect of macrofauna on porewater profiles and nutrient fluxes in the intertidal zone of the Humber estuary. Estuarine, Coastal and Shelf Science, 1999, 48(6): 683-699. DOI: 10. 1006/ecss. 1999.0479.

[12] Kraal P, Burton ED, Bush RT. Iron monosulfide accumulation and pyrite formation in eutrophic estuarine sediments. Geochimica et Cosmochimica Acta, 2013, 122: 75-88. DOI: 10.1016/j.gca.2013.08.013.

[13] Sheng YQ, Sun QY, Shi WJ et al. Geochemistry of reduced inorganic sulfur, reactive iron, and organic carbon in fluvial and marine surface sediment in the Laizhou Bay region, China. Environmental Earth Sciences, 2015, 74(2) : 1151-1160. DOI: $10.1007 / \mathrm{s} 12665-015-4101-8$.

[14] Sun QY, Sheng YQ, Yang J et al. Dynamic characteristics of sulfur, iron and phosphorus in coastal polluted sediments, North China. Environmental Pollution, 2016, 219: 588-595. DOI: 10.1016/j.envpol.2016.06.019.

[15] Werne J, Hollander D, Lyons T et al. Organic sulfur biogeochemistry: recent advances and future research directions. In: Amend JP, Edwards KJ, Lyons TW eds. Sulfur biogeochemistry-past and present: Boulder, Colorado, Geological Society of America Special 2004, 379: 117-134. DOI: 10.1130/0-8137-2379-5.135.

[16] Burton ED, Bush RT, Johnston SG et al. Sulfur biogeochemical cycling and novel Fe-S mineralization pathways in a tidally re-flooded wetland. Geochimica et Cosmochimica Acta, 2011, 75(12) : 3434-3451. DOI: 10.1016/j.gca.2011.03.020.

[17] Jiang M, Sheng YQ, Liu QQ et al. Conversion mechanisms between organic sulfur and inorganic sulfur in surface sediments in coastal rivers. Science of the Total Environment, 2021, 752: 141829. DOI: 10.1016/j.scitotenv.2020.141829.

[18] Morse JW, Rickard D. Chemical dynamics of sedimentary acid volatile sulfide. Environmental Science \& Technology, 2004, 38(7) : 131A-136A. DOI: 10.1021/es040447y.

[19] Jiang M, Zhao GQ, Li ZR et al. Distribution characteristics and relationship between reduced inorganic sulfur and reactive iron in core sediments outside the mouth of the Jiahe River in Yantai. Marine Sciences, 2018, 42 (8) : 90-97. DOI: 10. 11759/hykx20180313001. [姜明, 赵国强, 李兆再等. 烟台夹河口外柱状沉积物还原性无机硫、活性铁的变化特征 及其相互关系. 海洋科学, 2018, 42(8): 90-97.]

[20] Canfield DE, Thamdrup B. Fate of elemental sulfur in an intertidal sediment. FEMS Microbiology Ecology, 1996, 19(2): 95-103. DOI: 10.1016/0168-6496(95) 00083-6.

[21] Zhu MX, Shi XN, Yang GP et al. Formation and burial of pyrite and organic sulfur in mud sediments of the East China Sea inner shelf: Constraints from solid-phase sulfur speciation and stable sulfur isotope. Continental Shelf Research, $2013, \mathbf{5 4}$ : 24-36. DOI: 10.1016/j.csr.2013.01.002.

[22] Kang PG, Mitchell MJ, Mayer B et al. Isotopic evidence for determining the sources of dissolved organic sulfur in a forested catchment. Environmental Science \& Technology, 2014, 48(19) : 11259-11267. DOI: 10.1021/es502563n.

[23] Fike DA, Bradley AS, Rose CV. Rethinking the ancient sulfur cycle. Annual Review of Earth and Planetary Sciences, 2015, 43(1) : 593-622. DOI: 10.1146/annurev-earth-060313-054802.

[24] Brüchert V. Early diagenesis of sulfur in estuarine sediments: The role of sedimentary humic and fulvic acids. Geochimica et Cosmochimica Acta, 1998, 62(9) : 1567-1586. DOI: 10.1016/S0016-7037(98)00089-1.

[25] Anderson TF, Pratt LM. Isotopic evidence for the origin of organic sulfur and elemental sulfur in marine sediments. ACS Symposium Series. Washington, DC: American Chemical Society, 1995: 378-396. DOI: 10.1021/bk-1995-0612.ch021.

[26] Bottrell SH, Hatfield D, Bartlett R et al. Concentrations, sulfur isotopic compositions and origin of organosulfur compounds in pore waters of a highly polluted raised peatland. Organic Geochemistry, 2010, 41(1) : 55-62. DOI: 10.1016/j.orggeochem.2009.07.005.

[27] Shawar L, Halevy I, Said-Ahmad W et al. Dynamics of pyrite formation and organic matter sulfurization in organic-rich carbonate sediments. Geochimica et Cosmochimica Acta, 2018, 241: 219-239. DOI: 10.1016/j.gca.2018.08.048.

[28] Sela-Adler M, Said-Ahmad W, Sivan O et al. Isotopic evidence for the origin of dimethylsulfide and dimethylsulfoniopropionate-like compounds in a warm, monomictic freshwater lake. Environmental Chemistry, 2016, 13(2): 340. DOI: 10. 
1071/en15042.

[29] Habicht KS, Canfield DE. Sulfur isotope fractionation during bacterial sulfate reduction in organic-rich sediments. Geochimica et Cosmochimica Acta, 1997, 61(24) : 5351-5361. DOI: 10.1016/S0016-7037(97)00311-6.

[30] Brüchert V, Knoblauch C, Jørgensen BB. Controls on stable sulfur isotope fractionation during bacterial sulfate reduction in Arctic sediments. Geochimica et Cosmochimica Acta, 2001, 65(5) : 763-776. DOI: 10.1016/S0016-7037(00)00557-3.

[31] Aizenshtat Z, Amrani A. Significance of $\delta^{34} \mathrm{~S}$ and evaluation of its imprint on sedimentary organic matter: I. The role of reduced sulfur species in the diagenetic stage: A conceptual review. In: Hill RJ, Leventhal J et al eds. The geochemical society special publications. Amsterdam: Elsevier, 2004: 15-33. DOI: 10.1016/s1873-9881(04)80004-x.

[32] Sun LG, Xie ZQ, Zhao JL. A 3, 000-year record of penguin populations. Nature, 2000, 407 (6806) : 858. DOI: 10. $1038 / 35038163$.

[33] Chen YQ, Shen LL, Huang T et al. Transformation of sulfur species in lake sediments at Ardley Island and Fildes Peninsula, King George Island, Antarctic Peninsula. Science of the Total Environment, 2020, 703: 135591. DOI: 10.1016/j.scitotenv.2019.135591.

[34] Chen YQ, Ge JW, Huang T et al. Restriction of sulfate reduction on the bioavailability and toxicity of trace metals in Antarctic lake sediments. Marine Pollution Bulletin, 2020, 151: 110807. DOI: 10.1016/j.marpolbul.2019.110807.

[35] Berner RA, Scott MR, Thomlinson C. Carbonate alkalinity in the pore waters of anoxic marine sediments1. Limnology and Oceanography, 1970, 15(4) : 544-549. DOI: 10.4319/lo.1970.15.4.0544.

[36] Shen L, Huang T, Chen Y et al. Diverse transformations of sulfur in seabird-affected sediments revealed by microbial and stable isotope analysis. Manuscript submitted, 2021.

[37] Kaplan IR, Rittenberg SC. Microbiological fractionation of sulphur isotopes. Journal of General Microbiology, 1964, 34 : 195-212. DOI: 10.1099/00221287-34-2-195.

[38] Huang XL, Zhu MX, Chen LJ et al. Sources and formation mechanisms of organic sulfur in Jiaozhou Bay sediments. Acta Oceanologica Sinica, 2014, 36(6) : 50-57. [黄香利, 朱茂旭, 陈良进等. 胶州湾沉积物中有机硫来源及成因机制的 研究. 海洋学报: 中文版, 2014, 36(6) : 50-57.]

[39] Chen LJ, Zhu MX, Huang XL et al. Speciation of organic sulfur in inner shelf sediments of the East China Sea: Evidence from K-edge XANES spectroscopy. Geochimica, 2015, 44(1) : 61-70. DOI: 10.19700/j.0379-1726.2015.01.005. [陈良 进, 朱茂旭, 黄香利等. 东海内陆架沉积物中有机硫形态的 K 边 XANES 谱分析. 地球化学, 2015, 44(1): 61-70.]

[40] Werne JP, Lyons TW, Hollander DJ et al. Investigating pathways of diagenetic organic matter sulfurization using compound-specific sulfur isotope analysis. Geochimica et Cosmochimica Acta, 2008, 72(14) : 3489-3502. DOI: 10.1016/j. gca.2008.04.033.

[41] Li ST, Lin B, Zhou W. Soil organic sulfur mineralization in the presence of growing plants under aerobic or waterlogged conditions. Soil Biology and Biochemistry, 2001, 33(6) : 721-727. DOI: 10.1016/S0038-0717(00) 00205-4.

[42] Hartgers WA, Lòpez JF, Sinninghe Damsté JS et al. Sulfur-binding in recent environments: II. Speciation of sulfur and iron and implications for the occurrence of organo-sulfur compounds. Geochimica et Cosmochimica Acta, 1997, 61(22): 4769-4788. DOI: 10.1016/S0016-7037(97)00279-2.

[43] Cao A. The characteristic of reduced inorganic sulfur species in the Yangtze River Estuary and its environmental significance [Dissertation]. Shanghai : Fudan University, 2010. [曹爱丽. 长江口滨海沉积物中无机硫的形态特征及其环境 意义 [学位论文].上海: 复旦大学, 2010.]

[44] Brüchert V, Pratt LM. Contemporaneous early diagenetic formation of organic and inorganic sulfur in estuarine sediments from St. Andrew Bay, Florida, USA. Geochimica et Cosmochimica Acta, 1996, 60 (13) : 2325-2332. DOI: 10.1016/ 0016-7037 (96) 00087-7.

[45] Urban NR, Ernst K, Bernasconi S. Addition of sulfur to organic matter during early diagenesis of lake sediments. Geochimica et Cosmochimica Acta, 1999, 63(6) : 837-853. DOI: 10.1016/S0016-7037(98)00306-8.

[46] Filley TR, Freeman KH, Wilkin RT et al. Biogeochemical controls on reaction of sedimentary organic matter and aqueous sulfides in Holocene sediments of Mud Lake, Florida. Geochimica et Cosmochimica Acta, 2002, 66( 6) : 937-954. DOI: 10.1016/S0016-7037 ( 01 ) 00829-8.

[47] Wu SJ, Wang X, Ji QY et al. Iron-sulfur distribution and its environmental significance in three typical areas of western 
Lake Taihu. J Lake Sci, 2019, 31(4) : 950-960. DOI: 10.18307/2019.0408. [吴松峻, 汪旋, 季秋忆等. 太湖西岸典 型区域沉积物的硫铁分布特征及环境意义. 湖泊科学, 2019, 31(4) : 950-960.]

[48] Li YC, Yu S, Strong J et al. Are the biogeochemical cycles of carbon, nitrogen, sulfur, and phosphorus driven by the “FeIII-FeII redox wheel” in dynamic redox environments?. Journal of Soils and Sediments, 2012, 12(5) : 683-693. DOI: 10.1007/s11368-012-0507-z.

[49] Qin SS, Zhu MX, Yang GP et al. Atypical diagenesis of sulfur and iron in sediments of the river-dominated Bohai Sea (China). Journal of Marine Systems, 2019, 189: 116-126. DOI: 10.1016/j.jmarsys.2018.10.004.

[50] Oueslati W, Helali MA, Zaaboub N et al. Sulfide influence on metal behavior in a polluted southern Mediterranean lagoon: Implications for management. Environmental Science and Pollution Research, 2018, 25(3) : 2248-2264. DOI: 10.1007/ s11356-017-0529-6.

[51] Jørgensen BB, Findlay AJ, Pellerin A. The biogeochemical sulfur cycle of marine sediments. Frontiers in Microbiology, 2019, 10: 849. DOI: 10.3389/fmicb.2019.00849.

[52] Wu SJ, Zhao YP, Chen YY et al. Sulfur cycling in freshwater sediments: A cryptic driving force of iron deposition and phosphorus mobilization. Science of the Total Environment, 2019, 657: 1294-1303. DOI: 10. 1016/j. scitotenv. 2018. 12.161 .

[53] Sun LG, Emslie SD, Huang T et al. Vertebrate records in polar sediments: Biological responses to past climate change and human activities. Earth-Science Reviews, 2013, 126: 147-155. DOI : 10.1016/j.earscirev.2013.08.004.

[54] Zhu RB, Liu YS, Ma ED et al. Greenhouse gas emissions from penguin guanos and ornithogenic soils in coastal Antarctica: Effects of freezing-thawing cycles. Atmospheric Environment, 2009, 43(14) : 2336-2347. DOI: 10.1016/j.atmosenv. 2009.01.027. 